\title{
A fixed point problem under two constraint inequalities
}

\author{
Mohamed Jleli and Bessem Samet ${ }^{*}$
}

${ }^{\text {*Correspondence: }}$

bsamet@ksu.edu.sa

Department of Mathematics,

College of Science, King Saud

University, P.O. Box 2455, Riyadh,

11451, Saudi Arabia

\begin{abstract}
Let $(X, d)$ be a metric space. Suppose that the set $X$ is equipped with two partial orders $\preceq_{1}$ and $\preceq_{2}$. Let $T, A, B, C, D: X \rightarrow X$ be given operators. We provide sufficient conditions for the existence of a fixed point of $T$ satisfying the two constraint inequalities: $A x \preceq_{1} B x$ and $C x \preceq_{2} D x$.
\end{abstract}

Keywords: fixed point; constraint inequalities; partial order

\section{Introduction and basic definitions}

Recently there have been many developments concerning the existence of fixed points for operators defined in a metric space equipped with a partial order. This trend was initiated by Turinici [1]. Next, Ran and Reurings [2] extended the Banach contraction principle to continuous monotone operators defined in a partially ordered metric space. They also presented some applications to the existence of positive solutions to certain classes of nonlinear matrix equations. The result obtained in [2] was extended and generalized by many authors in different directions (see [3-11] and the references therein).

Let $(X, d)$ be a metric space. Suppose that the set $X$ is endowed with two partial orders $\preceq_{1}$ and $\preceq_{2}$. Let us consider five self-operators $T, A, B, C, D: X \rightarrow X$. In this paper, we are concerned with the following problem: Find $x \in X$ such that

$$
\left\{\begin{array}{l}
x=T x, \\
A x \preceq_{1} B x, \\
C x \preceq_{2} D x .
\end{array}\right.
$$

We obtain sufficient conditions for the existence of at least one solution to (1.1).

The following definitions will be used throughout the paper.

Definition 1.1 Let $X$ be a nonempty set. Let $\preceq$ be a binary relation on $X$. We say that $\preceq$ is a partial order on $X$ if the following conditions are satisfied:

(i) For every $x \in X$, we have $x \preceq x$.

(ii) For every $x, y, z \in X$, we have

$$
x \preceq y, \quad y \preceq z \quad \Longrightarrow \quad x \preceq z .
$$

(iii) For every $x, y \in X$, we have

$$
x \preceq y, \quad y \preceq x \Rightarrow x=y .
$$

(c) 2016 Jleli and Samet. This article is distributed under the terms of the Creative Commons Attribution 4.0 International License (http://creativecommons.org/licenses/by/4.0/), which permits unrestricted use, distribution, and reproduction in any medium, provided you give appropriate credit to the original author(s) and the source, provide a link to the Creative Commons license, and indicate if changes were made. 
Definition 1.2 Let $(X, d)$ be a metric space and $\preceq$ be a partial order on $X$. We say that the partial order $\preceq$ is $d$-regular if the following condition is satisfied: For every sequences $\left\{a_{n}\right\},\left\{b_{n}\right\} \subset X$, we have

$$
\lim _{n \rightarrow \infty} d\left(a_{n}, a\right)=\lim _{n \rightarrow \infty} d\left(b_{n}, b\right)=0, \quad a_{n} \preceq b_{n} \text { for all } n \quad \Longrightarrow \quad a \preceq b,
$$

where $(a, b) \in X \times X$.

Example 1.3 Let $(X,\|\cdot\|)$ be a Banach space and $P$ be a cone on $X$. Let us consider the partial order on $X$ defined by

$$
(x, y) \in X \times X, \quad x \preceq_{P} y \quad \Longleftrightarrow \quad y-x \in P .
$$

Consider the metric $d$ on $X$ defined by

$$
d(x, y)=\|x-y\|, \quad(x, y) \in X \times X .
$$

Then $\preceq_{P}$ is $d$-regular. In fact, suppose that $\left\{a_{n}\right\}$ and $\left\{b_{n}\right\}$ are two sequences in $X$ such that

$$
a_{n} \preceq_{P} b_{n} \quad \text { for all } n
$$

and

$$
\lim _{n \rightarrow \infty} d\left(a_{n}, a\right)=\lim _{n \rightarrow \infty} d\left(b_{n}, b\right)=0
$$

for some $(a, b) \in X \times X$. Since $b_{n}-a_{n} \in P$ for all $n$ and the sequence $\left\{b_{n}-a_{n}\right\}$ converges to $b-a$, the closure of the cone $P$ yields $b-a \in P$, that is, $a \preceq_{P} b$.

Definition 1.4 Let $X$ be a nonempty set endowed with two partial orders $\preceq_{1}$ and $\preceq_{2}$. Let $T, A, B, C, D: X \rightarrow X$ be given operators. We say that the operator $T$ is $\left(A, B, C, D, \preceq_{1}, \preceq_{2}\right)$ stable, if the following condition is satisfied:

$$
x \in X, \quad A x \preceq_{1} B x \quad \Longrightarrow \quad C T x \preceq_{2} D T x .
$$

Example 1.5 Let $X=\mathbb{R}$ and consider the standard order $\leq$ on $X$. Let $A, B, C, D: X \rightarrow X$ be the operators defined by

$$
\begin{aligned}
& A x=x, \quad B x=x^{2}, \quad C x=\exp (x), \\
& D x=\exp \left(x^{2}-2 x+2\right), \quad T x=x+1, \quad x \in \mathbb{R} .
\end{aligned}
$$

Then the operator $T$ is $(A, B, C, D, \leq, \leq)$-stable. In fact, let $x \in X$ be such that

$$
A x=x \leq B x=x^{2} .
$$

Then

$$
x+1 \leq x^{2}+1
$$


which yields

$$
\exp (x+1) \leq \exp \left(x^{2}+1\right)
$$

that is,

$$
C T x \leq D T x .
$$

Example 1.6 Let $X=\mathbb{R}^{2}$. We consider the two partial orders $\preceq_{1}$ and $\preceq_{2}$ on $X$ defined by

$$
(a, b),(c, d) \in X, \quad(a, b) \preceq_{1}(c, d) \quad \Longleftrightarrow \quad a \leq c, \quad b \leq d
$$

and

$$
(a, b),(c, d) \in X, \quad(a, b) \preceq_{2}(c, d) \quad \Longleftrightarrow \quad a \leq c, \quad b \geq d .
$$

Let us consider the operators $T, A, B, C, D: X \rightarrow X$ defined by

$$
\begin{aligned}
& T(a, b)=(b, a+1), \\
& A(a, b)=(a, 2 b), \\
& B(a, b)=(a, 3 b), \\
& C(a, b)=(|a|, b), \\
& D(a, b)=\left(\frac{(a+1)|a|}{|a|+1}, b-1\right)
\end{aligned}
$$

for all $(a, b) \in X$. We claim that $T$ is $\left(A, B, C, D, \preceq_{1}, \preceq_{2}\right)$-stable. In order to prove this claim, we take $x=(a, b) \in X$ such that

$$
A x \preceq_{1} B x,
$$

which is equivalent (from the definition of $\preceq_{1}$ ) to

$$
b \geq 0 .
$$

On the other hand, under the above inequality, we have

$$
C T x=C(b, a+1)=(|b|, a+1)=(b, a+1)
$$

and

$$
D T x=D(b, a+1)=\left(\frac{(b+1)|b|}{|b|+1}, a\right)=(b, a-1) .
$$

Clearly (from the definition of $\preceq_{2}$ ), we have

$$
C T x \preceq_{2} D T x .
$$

Then our claim is proved. 
Now, we are ready to state and prove our main result.

\section{Main result}

Let us denote by $\Phi$ the set of functions $\varphi:[0, \infty) \rightarrow[0, \infty)$ satisfying the following conditions:

$\left(\Phi_{1}\right) \varphi$ is a lower semi-continuous function.

$\left(\Phi_{2}\right) \varphi^{-1}(\{0\})=\{0\}$.

Our main result in this paper is giving by the following theorem.

Theorem 2.1 Let $(X, d)$ be a complete metric space endowed with two partial orders $\preceq_{1}$ and $\preceq_{2}$. Let $T, A, B, C, D: X \rightarrow X$ be given operators. Suppose that the following conditions are satisfied:

(i) $\preceq_{i}$ is $d$-regular, $i=1,2$.

(ii) $A, B, C$, and $D$ are continuous.

(iii) There exists $x_{0} \in X$ such that

$$
A x_{0} \preceq_{1} B x_{0} .
$$

(iv) $T$ is $\left(A, B, C, D, \preceq_{1}, \preceq_{2}\right)$-stable.

(v) $T$ is $\left(C, D, A, B, \preceq_{2}, \preceq_{1}\right)$-stable.

(vi) There exists $\varphi \in \Phi$ such that

$$
A x \preceq_{1} B x, \quad C y \preceq_{2} D y \quad \Longrightarrow \quad d(T x, T y) \leq d(x, y)-\varphi(d(x, y)) .
$$

Then:

(I) The sequence $\left\{T^{n} x_{0}\right\}$ converges to some $x^{*} \in X$ satisfying

$$
A x^{*} \preceq_{1} B x^{*} \text { and } C x^{*} \preceq_{2} D x^{*} .
$$

(II) The point $x^{*} \in X$ is a solution to (1.1).

Proof Let us prove (I). Let $x_{0} \in X$ be such that

$$
A x_{0} \preceq_{1} B x_{0} .
$$

Such a point exists from (iii). Let us consider the sequence $\left\{x_{n}\right\} \subset X$ defined by

$$
x_{n}=T^{n} x_{0}, \quad n=0,1,2, \ldots
$$

Since $T$ is $\left(A, B, C, D, \preceq_{1}, \preceq_{2}\right)$-stable (see (iv)), we have

$$
A x_{0} \preceq_{1} B x_{0} \quad \Longrightarrow \quad C T x_{0} \preceq_{2} D T x_{0},
$$

that is,

$$
C x_{1} \preceq_{2} D x_{1} .
$$


Then we have

$$
A x_{0} \preceq_{1} B x_{0} \text { and } C x_{1} \preceq_{2} D x_{1} \text {. }
$$

Since $T$ is $\left(C, D, A, B, \preceq_{2}, \preceq_{1}\right)$-stable (see (v)),

$$
C x_{1} \preceq_{2} D x_{1} \quad \Longrightarrow \quad A T x_{1} \preceq_{1} B T x_{1}
$$

that is,

$$
A x_{2} \preceq_{1} B x_{2} .
$$

Again, $T$ is $\left(A, B, C, D, \preceq_{1}, \preceq_{2}\right)$-stable; this yields

$$
A x_{2} \preceq_{1} B x_{2} \quad \Longrightarrow \quad C T x_{2} \preceq_{2} D T x_{2},
$$

that is,

$$
C x_{3} \preceq_{2} D x_{3} .
$$

Thus we have

$$
A x_{2} \preceq_{1} B x_{2} \text { and } C x_{3} \preceq_{2} D x_{3} \text {. }
$$

By induction, we get

$$
A x_{2 n} \preceq_{1} B x_{2 n} \quad \text { and } \quad C x_{2 n+1} \preceq_{2} D x_{2 n+1}, \quad n=0,1,2, \ldots
$$

Using (2.1) and (vi), by symmetry, we have

$$
d\left(x_{n+1}, x_{n}\right)=d\left(T x_{n}, T x_{n-1}\right) \leq d\left(x_{n-1}, x_{n}\right)-\varphi\left(d\left(x_{n-1}, x_{n}\right)\right), \quad n=1,2,3, \ldots,
$$

which yields

$$
d\left(x_{n+1}, x_{n}\right) \leq d\left(x_{n}, x_{n-1}\right), \quad n=1,2,3, \ldots
$$

Then $\left\{d\left(x_{n+1}, x_{n}\right)\right\}$ is a decreasing sequence of positive numbers. Therefore, there exists some $r \geq 0$ such that

$$
\lim _{n \rightarrow \infty} d\left(x_{n+1}, x_{n}\right)=r
$$

From (2.2), we have

$$
d\left(x_{n+1}, x_{n}\right)+\varphi\left(d\left(x_{n-1}, x_{n}\right)\right) \leq d\left(x_{n-1}, x_{n}\right), \quad n=1,2,3, \ldots,
$$

which yields

$$
\liminf _{n \rightarrow \infty}\left(d\left(x_{n+1}, x_{n}\right)+\varphi\left(d\left(x_{n-1}, x_{n}\right)\right)\right) \leq \liminf _{n \rightarrow \infty} d\left(x_{n-1}, x_{n}\right) .
$$


Using (2.3) and the lower semi-continuity of $\varphi$, we obtain

$$
r+\varphi(r) \leq r
$$

which implies that

$$
r \in \varphi^{-1}(\{0\}) .
$$

Since $\varphi^{-1}(\{0\})=\{0\}$, we get $r=0$, i.e.,

$$
\lim _{n \rightarrow \infty} d\left(x_{n+1}, x_{n}\right)=0
$$

Now, we show that $\left\{x_{n}\right\}$ is a Cauchy sequence in $(X, d)$. Suppose that $\left\{x_{n}\right\}$ is not a Cauchy sequence. Then there exists some $\varepsilon>0$ for which we find two sequences of positive integers $\{m(k)\}$ and $\{n(k)\}$ such that, for all positive integers $k$,

$$
n(k)>m(k)>k, \quad d\left(x_{m(k)}, x_{n(k)}\right) \geq \varepsilon, \quad d\left(x_{m(k)}, x_{n(k)-1}\right)<\varepsilon .
$$

From (2.5), we have

$$
\begin{aligned}
\varepsilon & \leq d\left(x_{m(k)}, x_{n(k)}\right) \\
& \leq d\left(x_{m(k)}, x_{n(k)-1}\right)+d\left(x_{n(k)-1}, x_{n(k)}\right) \\
& <\varepsilon+d\left(x_{n(k)-1}, x_{n(k)}\right) .
\end{aligned}
$$

Then, for all $k$, we have

$$
\varepsilon \leq d\left(x_{m(k)}, x_{n(k)}\right)<\varepsilon+d\left(x_{n(k)-1}, x_{n(k)}\right) .
$$

Passing to the limit as $k \rightarrow \infty$ and using (2.4), we get

$$
\lim _{k \rightarrow \infty} d\left(x_{m(k)}, x_{n(k)}\right)=\varepsilon
$$

On the other hand, we have

$$
\left|d\left(x_{n(k)+1}, x_{m(k)}\right)-d\left(x_{m(k)}, x_{n(k)}\right)\right| \leq d\left(x_{n(k)+1}, x_{n(k)}\right) \rightarrow 0 \quad \text { as } k \rightarrow \infty(\text { from (2.4)) } .
$$

Then from (2.6), we have

$$
\lim _{k \rightarrow \infty} d\left(x_{n(k)+1}, x_{m(k)}\right)=\varepsilon
$$

We have also

$$
\left|d\left(x_{m(k)}, x_{n(k)}\right)-d\left(x_{n(k)}, x_{m(k)-1}\right)\right| \leq d\left(x_{m(k)-1}, x_{m(k)}\right) \rightarrow 0 \quad \text { as } k \rightarrow \infty \text { (from (2.4)). }
$$

Then from (2.6), we have

$$
\lim _{k \rightarrow \infty} d\left(x_{n(k)}, x_{m(k)-1}\right)=\varepsilon
$$


Similarly,

$$
\left|d\left(x_{n(k)+1}, x_{m(k)}\right)-d\left(x_{n(k)+1}, x_{m(k)+1}\right)\right| \leq d\left(x_{m(k)}, x_{m(k)+1}\right) \rightarrow 0 \quad \text { as } k \rightarrow \infty(\text { from }(2.4)) .
$$

Then from (2.7), we have

$$
\lim _{k \rightarrow \infty} d\left(x_{n(k)+1}, x_{m(k)+1}\right)=\varepsilon
$$

Observe that, for all $k$, there exists a positive integer $0 \leq i(k) \leq 1$ such that

$$
n(k)-m(k)+i(k) \equiv 1(2)
$$

From (2.1), for all $k>1$, we have

$$
A x_{n(k)} \preceq_{1} B x_{n(k)} \text { and } C x_{m(k)-i(k)} \preceq_{2} D x_{m(k)-i(k)}
$$

or

$$
A x_{m(k)-i(k)} \preceq_{1} B x_{m(k)-i(k)} \quad \text { and } \quad C x_{n(k)} \preceq_{2} D x_{n(k)} .
$$

Then from (vi), by symmetry, we have

$$
d\left(T x_{n(k)}, T x_{m(k)-i(k)}\right) \leq d\left(x_{n(k)}, x_{m(k)-i(k)}\right)-\varphi\left(d\left(x_{n(k)}, x_{m(k)-i(k)}\right)\right), \quad k>1,
$$

that is,

$$
d\left(x_{n(k)+1}, x_{m(k)-i(k)+1}\right) \leq d\left(x_{n(k)}, x_{m(k)-i(k)}\right)-\varphi\left(d\left(x_{n(k)}, x_{m(k)-i(k)}\right)\right), \quad k>1 .
$$

Set

$$
\Lambda=\{k>1: i(k)=0\} \quad \text { and } \quad \Delta=\{k>1: i(k)=1\} .
$$

We consider two cases.

- Case $1 .|\Lambda|=\infty$.

From (2.10), we get

$$
d\left(x_{n(k)+1}, x_{m(k)+1}\right)+\varphi\left(d\left(x_{n(k)}, x_{m(k)}\right)\right) \leq d\left(x_{n(k)}, x_{m(k)}\right), \quad k \in \Lambda,
$$

which gives us

$$
\liminf _{k \rightarrow \infty}\left(d\left(x_{n(k)+1}, x_{m(k)+1}\right)+\varphi\left(d\left(x_{n(k)}, x_{m(k)}\right)\right)\right) \leq \liminf _{k \rightarrow \infty} d\left(x_{n(k)}, x_{m(k)}\right) .
$$

Using (2.9), (2.6), and the lower semi-continuity of $\varphi$, we obtain

$$
\varepsilon+\varphi(\varepsilon) \leq \varepsilon
$$


which yields

$$
\varepsilon \in \varphi^{-1}(\{0\}) .
$$

Since $\varphi^{-1}(\{0\})=\{0\}$, we get $\varepsilon=0$, which is a contradiction with $\varepsilon>0$.

- Case $2 .|\Lambda|<\infty$.

In this case, we have $|\Delta|=\infty$. Moreover, from (2.10), we have

$$
d\left(x_{n(k)+1}, x_{m(k)}\right)+\varphi\left(d\left(x_{n(k)}, x_{m(k)-1}\right)\right) \leq d\left(x_{n(k)}, x_{m(k)-1}\right), \quad k \in \Delta,
$$

which gives us

$$
\liminf _{k \rightarrow \infty}\left(d\left(x_{n(k)+1}, x_{m(k)}\right)+\varphi\left(d\left(x_{n(k)}, x_{m(k)-1}\right)\right)\right) \leq \liminf _{k \rightarrow \infty} d\left(x_{n(k)}, x_{m(k)-1}\right) .
$$

Using (2.7), (2.8), and the lower semi-continuity of $\varphi$, we obtain

$$
\varepsilon+\varphi(\varepsilon) \leq \varepsilon
$$

which yields $\varepsilon \in \varphi^{-1}(\{0\})=\{0\}$, that is, a contradiction with $\varepsilon>0$.

Therefore, we deduce that $\left\{x_{n}\right\}$ is a Cauchy sequence in $(X, d)$. Since $(X, d)$ is complete, there exists some $x^{*} \in X$ such that

$$
\lim _{n \rightarrow \infty} d\left(x_{n}, x^{*}\right)=0 .
$$

On the other hand, from (2.1), we have

$$
A x_{2 n} \preceq_{1} B x_{2 n}, \quad n=0,1,2, \ldots
$$

Using the continuity of $A$ and $B$, it follows from (2.11) that

$$
\lim _{n \rightarrow \infty} d\left(A x_{2 n}, A x^{*}\right)=\lim _{n \rightarrow \infty} d\left(B x_{2 n}, B x^{*}\right)=0 .
$$

Since $\preceq_{1}$ is $d$-regular, we get

$$
A x^{*} \preceq_{1} B x^{*} .
$$

Similarly, from (2.1), we have

$$
C x_{2 n+1} \preceq_{2} D x_{2 n+1}, \quad n=0,1,2, \ldots
$$

Using the continuity of $C$ and $D$, it follows from (2.11) that

$$
\lim _{n \rightarrow \infty} d\left(C x_{2 n+1}, C x^{*}\right)=\lim _{n \rightarrow \infty} d\left(D x_{2 n+1}, D x^{*}\right)=0 .
$$

Since $\preceq_{2}$ is $d$-regular, we get

$$
C x^{*} \preceq_{2} D x^{*}
$$

Thus we proved (I). 
Now, let us prove (II). Using (2.12), (2.13), (2.1), and (vi), we have

$$
\begin{aligned}
d\left(T x^{*}, x^{*}\right) & \leq d\left(T x^{*}, T x_{2 n}\right)+d\left(x_{2 n+1}, x^{*}\right) \\
& \leq d\left(x^{*}, x_{2 n}\right)-\varphi\left(d\left(x^{*}, x_{2 n}\right)\right)+d\left(x_{2 n+1}, x^{*}\right), \quad n=0,1,2, \ldots
\end{aligned}
$$

that is,

$$
d\left(T x^{*}, x^{*}\right)+\varphi\left(d\left(x^{*}, x_{2 n}\right)\right) \leq d\left(x^{*}, x_{2 n}\right)+d\left(x_{2 n+1}, x^{*}\right), \quad n=0,1,2, \ldots
$$

Then

$$
\liminf _{n \rightarrow \infty}\left(d\left(x^{*}, x^{*}\right)+\varphi\left(d\left(x^{*}, x_{2 n}\right)\right)\right) \leq \liminf _{n \rightarrow \infty}\left(d\left(x^{*}, x_{2 n}\right)+d\left(x_{2 n+1}, x^{*}\right)\right) .
$$

Using the lower semi-continuity of $\varphi$, the fact that $\varphi(0)=0$, and (2.11), we obtain

$$
d\left(x^{*}, T x^{*}\right)=0,
$$

which yields

$$
T x^{*}=x^{*}
$$

Therefore, from (2.12), (2.13), and (2.14), we deduce that $x^{*} \in X$ is a solution to (1.1). This ends the proof.

In the next section, we present some consequences following from Theorem 2.1.

\section{Some consequences}

\subsection{A fixed point problem under one constraint equality}

Here, we are concerned with the following problem: Find $x \in X$ such that

$$
\left\{\begin{array}{l}
x=T x \\
A x=B x
\end{array}\right.
$$

where $T, A, B: X \rightarrow X$ are given operators and $(X, d)$ is a metric space endowed with a certain partial order $\preceq$. Observe that (3.1) is equivalent to (1.1) with

$$
\preceq_{1}=\preceq_{2}=\preceq, \quad C=B \quad \text { and } \quad D=A .
$$

Then from Theorem 2.1, we obtain the following result.

Corollary 3.1 Let $(X, d)$ be a complete metric space endowed with a certain partial order $\preceq$. Let $T, A, B: X \rightarrow X$ be three given operators. Suppose that the following conditions are satisfied:

(i) $\preceq$ is d-regular.

(ii) $A$ and $B$ are continuous. 
(iii) There exists $x_{0} \in X$ such that

$$
A x_{0} \preceq B x_{0} .
$$

(iv) For all $x \in X$, we have

$$
A x \preceq B x \quad \Longrightarrow \quad B T x \preceq A T x .
$$

(v) For all $x \in X$, we have

$$
B x \preceq A x \quad \Longrightarrow \quad A T x \preceq B T x .
$$

(vi) There exists $\varphi \in \Phi$ such that

$$
A x \preceq B x, \quad B y \preceq A y \quad \Longrightarrow \quad d(T x, T y) \leq d(x, y)-\varphi(d(x, y)) .
$$

Then:

(I) The sequence $\left\{T^{n} x_{0}\right\}$ converges to some $x^{*} \in X$ satisfying $A x^{*}=B x^{*}$.

(II) The point $x^{*} \in X$ is a solution to (3.1).

\subsection{A common fixed point problem}

Let us consider the following problem: Find $x \in X$ such that

$$
\left\{\begin{array}{l}
x=T x, \\
x=B x,
\end{array}\right.
$$

where $T, B: X \rightarrow X$ are given operators and $(X, d)$ is a metric space endowed with a certain partial order $\preceq$. Observe that (3.2) is equivalent to (3.1) with $A=I_{X}$, the identity mapping on $X$. So, take $A=I_{X}$ in Corollary 3.1, We obtain the following result.

Corollary 3.2 Let $(X, d)$ be a complete metric space endowed with a certain partial order $\preceq$. Let $T, B: X \rightarrow X$ be two given operators. Suppose that the following conditions are satisfied:

(i) $\preceq$ is $d$-regular.

(ii) $B$ is continuous.

(iii) There exists $x_{0} \in X$ such that

$$
x_{0} \preceq B x_{0} .
$$

(iv) For all $x \in X$, we have

$$
x \preceq B x \quad \Longrightarrow \quad B T x \preceq T x .
$$

(v) For all $x \in X$, we have

$$
B x \preceq x \quad \Longrightarrow \quad T x \preceq B T x .
$$


(vi) There exists $\varphi \in \Phi$ such that

$$
x \preceq B x, \quad B y \preceq y \quad \Longrightarrow \quad d(T x, T y) \leq d(x, y)-\varphi(d(x, y)) .
$$

Then:

(I) The sequence $\left\{T^{n} x_{0}\right\}$ converges to some $x^{*} \in X$ satisfying $x^{*}=B x^{*}$.

(II) The point $x^{*} \in X$ is a solution to (3.2).

Next, we present an example that illustrates the above result.

Example 3.3 Let $X \subset \mathbb{R}^{2}$ be the set defined by

$$
X=\{(4,0),(0,0),(6,0),(3,0),(3,1)\}
$$

Let $\preceq$ be the partial order on $X$ defined by

$$
(x, y),(z, w) \in X, \quad(x, y) \preceq(z, w) \quad \Longleftrightarrow \quad x \leq z, \quad y \leq w .
$$

We endow $X$ with the metric $d$ defined by

$$
d((x, y),(z, w))=\sqrt{(x-z)^{2}+(y-w)^{2}}, \quad(x, y),(z, w) \in X .
$$

Let $T, B: X \rightarrow X$ be the mappings defined by

$$
\left\{\begin{array} { l } 
{ T ( 4 , 0 ) = ( 4 , 0 ) , } \\
{ T ( 0 , 0 ) = ( 6 , 0 ) , } \\
{ T ( 6 , 0 ) = ( 3 , 0 ) , } \\
{ T ( 3 , 0 ) = ( 4 , 0 ) , } \\
{ T ( 3 , 1 ) = ( 0 , 0 ) }
\end{array} \quad \text { and } \quad \left\{\begin{array}{l}
B(4,0)=(4,0), \\
B(0,0)=(3,0), \\
B(6,0)=(0,0), \\
B(3,0)=(6,0), \\
B(3,1)=(4,0) .
\end{array}\right.\right.
$$

Observe that

$$
u \in X, \quad u \preceq B u \quad \Longleftrightarrow \quad u \in\{(4,0),(0,0),(3,0)\}
$$

However,

$$
v \in X, \quad B v \preceq v \Longleftrightarrow v \in\{(4,0),(6,0)\} .
$$

Let $u \in X$ be such that $u \preceq B u$.

For $u=(4,0)$, we have

$$
B T u=B(4,0)=(4,0)=T u .
$$

For $u=(0,0)$, we have

$$
B T u=B(6,0)=(0,0) \preceq(6,0)=T u .
$$


For $u=(3,0)$, we have

$$
B T u=B(4,0)=(4,0)=T u
$$

Then we have

$$
u \in X, \quad u \preceq B u \quad \Longrightarrow \quad B T u \preceq T u .
$$

Let $v \in X$ be such that $B v \preceq v$.

For $v=(4,0)$, we have

$$
T \nu=T(4,0)=(4,0)=B T \nu .
$$

For $v=(6,0)$, we have

$$
T v=T(6,0)=(3,0)=(6,0)=B T \text {. }
$$

Then we have

$$
v \in X, \quad B v \preceq v \quad \Longrightarrow \quad T v \preceq B T v .
$$

Now, let $(u, v) \in X \times X$ be such that

$$
u \preceq B u \quad \text { and } \quad B v \preceq v .
$$

We have

$$
\begin{array}{r}
(u, v) \in\{((4,0),(4,0)),((4,0),(6,0)),((0,0),(4,0)), \\
((0,0),(6,0)),((3,0),(4,0)),((3,0),(6,0))\} .
\end{array}
$$

For $(u, v)=((4,0),(4,0))$, we have

$$
d(T u, T v)=d((4,0),(4,0))=0 .
$$

For $(u, v)=((4,0),(6,0))$, we have

$$
d(T u, T v)=d((4,0),(3,0))=1=\frac{d(u, v)}{2}
$$

For $(u, v)=((0,0),(4,0))$, we have

$$
d(T u, T v)=d((6,0),(4,0))=2=\frac{d(u, v)}{2} .
$$

For $(u, v)=((0,0),(6,0))$, we have

$$
d(T u, T v)=d((6,0),(3,0))=3=\frac{d(u, v)}{2}
$$


For $(u, v)=((3,0),(4,0))$, we have

$$
d(T u, T v)=d((4,0),(4,0))=0 .
$$

For $(u, v)=((3,0),(6,0))$, we have

$$
d(T u, T v)=d((4,0),(3,0))=1<\frac{3}{2}=\frac{d(u, v)}{2} .
$$

Then we have

$$
u \preceq B u, \quad B v \preceq v \quad \Longrightarrow \quad d(T u, T v) \leq d(u, v)-\varphi(d(u, v)),
$$

where $\varphi(t)=\frac{t}{2}, t \geq 0$. So, all the required conditions of Corollary 3.2 are satisfied. Observe that $x^{*}=(4,0)$ is a common fixed point of $T$ and $B$. Observe also that

$$
d(T(4,0), T(3,1))=d((4,0),(0,0))=4>\sqrt{2}=d((4,0),(3,1)),
$$

which shows that $T$ is not a contraction on $X$.

Taking $B=T$ in Corollary 3.2, we obtain the following fixed point result.

Corollary 3.4 Let $(X, d)$ be a complete metric space endowed with a certain partial order $\preceq$. Let $T: X \rightarrow X$ be a given operator. Suppose that the following conditions are satisfied:

(i) $\preceq$ is d-regular.

(ii) $T$ is continuous.

(iii) There exists $x_{0} \in X$ such that

$$
x_{0} \preceq T x_{0} .
$$

(iv) For all $x \in X$, we have

$$
x \preceq T x \quad \Longrightarrow \quad T^{2} x \preceq T x
$$

(v) For all $x \in X$, we have

$$
T x \preceq x \quad \Longrightarrow \quad T x \preceq T^{2} x
$$

(vi) There exists $\varphi \in \Phi$ such that

$$
x \preceq T x, \quad T y \preceq y \quad \Longrightarrow \quad d(T x, T y) \leq d(x, y)-\varphi(d(x, y)) .
$$

Then the sequence $\left\{T^{n} x_{0}\right\}$ converges to a fixed point of $T$.

\section{Conclusion}

In this paper, we obtained sufficient conditions for the existence of a fixed point of a certain operator under two constraint inequalities with respect to two partial orders. The used 
technique can also be adapted for any finite number of constraint inequalities and other contractive conditions. An interesting question is the existence of a best proximity point of a certain operator under constraint inequalities. Such a question will be studied in a future work.

\section{Competing interests}

The authors declare that they have no competing interests.

\section{Authors' contributions}

All authors contributed equally and significantly in writing this article. All authors read and approved the final manuscript.

\section{Acknowledgements}

The authors extend their appreciation to Distinguished Scientist Fellowship Program (DSFP) at King Saud University (Saudi Arabia).

Received: 3 December 2015 Accepted: 25 January 2016 Published online: 03 February 2016

\section{References}

1. Turinici, M: Abstract comparison principles and multivariable Gronwall-Bellman inequalities. J. Math. Anal. Appl. 117, 100-127 (1986)

2. Ran, ACM, Reurings, MCB: A fixed point theorem in partially ordered sets and some applications to matrix equations. Proc. Am. Math. Soc. 132, 1435-1443 (2004)

3. Agarwal, RP, El-Gebeily, MA, O’Regan, D: Generalized contractions in partially ordered metric spaces. Appl. Anal. 87, 1-8 (2008)

4. Altun, I, Simsek, H: Some fixed point theorems on ordered metric spaces and application. Fixed Point Theory Appl. 2010, Article ID 621492 (2010)

5. Harjani, J, Sadarangani, K: Fixed point theorems for weakly contractive mappings in partially ordered sets. Nonlinear Anal. 71, 3403-3410 (2009)

6. Karapinar, E: Couple fixed point theorems for nonlinear contractions in cone metric spaces. Comput. Math. Appl. 59, 3656-3668 (2010)

7. Lakshmikantham, V, Ćirić, L: Coupled fixed point theorems for nonlinear contractions in partially ordered metric spaces. Nonlinear Anal. 70, 4341-4349 (2009)

8. Nieto, JJ, Rodriguez-López, R: Contractive mapping theorems in partially ordered sets and applications to ordinary differential equations. Order 22(3), 223-239 (2005)

9. Samet, B: Fixed point for $\alpha-\psi$ contractive mappings with an application to quadratic integral equations. Electron. J. Differ. Equ. 2014, 152 (2014)

10. Samet, B, Turinici, M: Fixed point theorems on a metric space endowed with an arbitrary binary relation and applications. Commun. Math. Anal. 13(2), 82-97 (2012)

11. Samet, B, Vetro, C: Coupled fixed point theorems for multi-valued nonlinear contraction mappings in partially ordered metric spaces. Nonlinear Anal. 74, 4260-4268 (2011)

\section{Submit your manuscript to a SpringerOpen ${ }^{\odot}$ journal and benefit from:}

- Convenient online submission

Rigorous peer review

- Immediate publication on acceptance

- Open access: articles freely available online

- High visibility within the field

- Retaining the copyright to your article 